March/April 1992; 33:298-303.) (Reprints: Dr. M. Duchowny Comprehensive Epilepsy Center, Miami Children's Hospital, 3200 S.W. 60 Court, Miami, FL 33155.)

COMMENT. Anterior temporal lobectomy tailored according to the extent of the lesion and epileptogenic field may be beneficial in preadolescent children with medically refractory seizures. Compared to adults, children undergoing temporal lobectomy have a higher incidence of abnormalities of neurogenesis and lesions that are poorly circumscribed and often widespread.

\title{
INTRACRANIAL ARACHNOIDAL CYSTS
}

Of 28 cases of intracranial arachnoidal cysts reported from the Clinica Las Condes, Santiago, Chile, 17 were located in the middle cranial fossa, 3 in the quadrigeminal cistern, 2 were parasagital, 3 suprasellar and 3 were in the posterior fossa. Of $23(82 \%)$ treated surgically, 3 received craniotomy with fenestrations, 8 had shunts and 15 were treated by the insertion of cystoperitoneal drainage with excellent results. Twenty-one of the 22 treated cases are asymptomatic and leading a normal life. MRI or CT follow-up showed disappearance of cysts in 14 and marked reduction in size in 7 . (Basauri L, Selman JM. Intracranial arachnoidal cysts. Child's Nerv Syst March 1992; $\underline{8}: 101-104$.) (Reprints: Dr. L. Basauri, Neurosurgical Unit, Clinica Las Condes, Casilla 27014, Santiago, Chile.)

COMMENT. The diagnosis of intracranial arachnoidal cysts and reports in the literature have become more frequent since the routine use of neuroimaging. The danger of intracystic hemorrhage with a high possibility of secondary hydrocephalus following head injury has been quoted as a reason for surgical intervention, as a preventive measure in some cases. See Ped Neur Briefs Oct 1991, $\underline{5}: 80$ for a report of 67 childhood arachnoidal cysts treated surgically and 1 report of spontaneous disappearance of a cyst. Small or medium sized cysts without clinical manifestations are not usually treated but are monitored carefully.

\section{UNBOUND PHENYTOIN AND VALPROATE MONITORING}

All antiepileptic drug blood level determinations at the University of Virginia Medical Center were analyzed over a 13 month period. Those values with unbound fractions outside the expected range and discordance between unbound and total levels were compared to seizure control and occurrence of side effects. Unbound phenytoin (PHT) levels were clinically significant (outside the expected range, a discordant ratio of unbound to total level, and seizures or side effects necessitating dosage change) in 61 of 254 determinations $(24 \%)$ and 44 of 155 patients $(28 \%)$. The clinical significance involved current seizures for 52 values and side effects for 24 (both for 15 ). The frequency of clinically significant values was similar with PHT monotherapy $(20 \%)$ and polytherapy $(27 \%)$. The rates of current seizures and side effects were also similar in these 2 subgroups. For valproate (VPA), $15 \%$ of unbound values from $18 \%$ of patients were clinically significant with regard to current seizures and side effects and the rate was similar for monotherapy and polytherapy. There were no clinically significant unbound 
levels for carbamazepine (Lenn NJ, Robertson M. Clinical utility of unbound antiepileptic drug blood levels in the management of epilepsy. Neurology May 1992; 42:988-990). (Reprints: Dr. Nicholas J. Lenn, Department of Neurology, Health Sciences Center T12-020, SUNY, Stony Brook, NY 11794-8121.)

COMMENT. These data suggest that some patients receiving phenytoin or valproate have unbound drug levels and ratios that may add information of importance to improvement of seizure control and reduction of side effects. The authors suggest that patients receiving PHT as mono- or polytherapy be monitored with either unbound blood level measurements only or have unbound blood levels regardless of the total level if there is an unresolved clinical problem. The expected unbound fraction of PHT is $8-12 \%$.

In a 17-year-old girl with thrombotic thrombocytopenia purpura who was treated by plasmapheresis the free and total concentrations of phenytoin used for the treatment of a coincident seizure disorder were examined in the patient's serum and in the plasma removed by plasmapheresis. The free concentration was similar in both the plasma removed and in the patient's serum. Plasmapheresis did not significantly alter the serum concentration of phenytoin and dosage adjustments of phenytoin are therefore unnecessary when single volume exchanges are performed. (Tobias JD et al. Removal of phenytoin by plasmapheresis in a patient with thrombotic thrombocytopenia purpura. Clin Pediatrics Feb 1992; 31:105-108.) These authors question the value of plasmapheresis and single volume exchange as an appropriate therapeutic intervention for phenytoin overdose. (Correspondence: Dr. Tobias, Vanderbilt University, Department of Pediatrics, Division of Pediatric Critical Care, Medical Center North T-0118, Nashville, TN 37232.)

\section{TOXIC DISORDERS}

\section{ASPARTAME AND ABSENCE SEIZURES}

The effect of aspartame on epilepsy was studied in 10 children with previously untreated generalized absence seizures using a double blind challenge of $40 \mathrm{mg} / \mathrm{kg}$ aspartame as a single dose at the Children's Hospital, Halifax, Canada. Ambulatory cassette recording of the EEG and quantification of numbers and length of spike wave discharges on 2 consecutive days showed that the number of seconds spent in spike wave discharge per hour of recording was significantly increased following aspartame compared with the control day when the children received sucrose. (Camfield PR et al. Aspartame exacerbates EEG spike-wave discharge in children with generalized absence epilepsy: a double-blind controlled study. Neurology May 1992; 42:1000-1003.) (Reprints: Dr. Peter Camfield, IWK Children's Hospital, Box 3070, Halifax, Nova Scotia, Canada B3J 3G9.)

COMMENT. Anecdotal claims that aspartame may exacerbate epilepsy appear to have scientific validity based on this EEG double blind controlled study in 10 children with absence seizures. 